\title{
Frequent mutation of hypoxia-related genes in persistent pulmonary hypertension of the newborn
}

Mingbang Wang ${ }^{1 * \dagger} \mathbb{D}$, Deyi Zhuang ${ }^{2 \dagger}$, Mei Mei ${ }^{3+}$, Haiyan $\mathrm{Ma}^{4}$, Zixiu Li ${ }^{5}$, Fusheng He ${ }^{6}$, Guoqiang Cheng ${ }^{7,8}$, Guang Lin $^{4^{*}}$ and Wenhao Zhou ${ }^{1 *}$

\begin{abstract}
Aims: Persistent pulmonary hypertension of the newborn (PPHN) is characterized by sustained high levels of pulmonary vascular resistance after birth with etiology unclear; Arterial blood oxygen saturation of Tibetan newborns at high latitudes is higher than that of Han newborns at low latitudes, suggesting that genetic adaptation may allow sufficient oxygen to confer Tibetan populations with resistance to pulmonary hypertension; We have previously identified genetic factors related to PPHN through candidate gene sequencing; In this study, we first performed whole exome sequencing in PPHN patients to screen for genetic-related factors.

Methods and results: In this two-phase genetic study, we first sequenced the whole exome of 20 Tibetan PPHN patients and compared it with the published genome sequences of 50 healthy high-altitude Tibetanshypoxia-related genes, a total of 166 PPHN-related variants were found, of which 49\% were from 43 hypoxia-related genes; considering many studies have shown that the differences in the genetic background between Tibet and Han are characterized by hypoxia-related genetic polymorphisms, so it is necessary to further verify whether the association between hypoxiarelated variants and PPHN is independent of high-altitude life. During the validation phase, 237 hypoxia-related genes were sequenced in another 80 Han PPHN patients living in low altitude areas, including genes at the discovery stage and known hypoxia tolerance, of which 413 variants from 127 of these genes were shown to be significantly associated with PPHN.hypoxia-related genes.
\end{abstract}

Conclusions: Our results indicates that the association of hypoxia-related genes with PPHN does not depend on highaltitude life, at the same time, 21 rare mutations associated with PPHN were also found, including three rare variants of the tubulin tyrosine ligase-like family member 3 gene (TTLL3:p.E317K, TTLL3:p.P7775) and the integrin subunit alpha M gene (ITGAM:p.E1071D). These novel findings provide important information on the genetic basis of PPHN.

Keywords: Persistent pulmonary hypertension of the newborn, Whole exome sequencing, Target region sequencing, Hypoxia tolerance

\footnotetext{
*Correspondence: Mingbang.wang.bgi@qq.com; linguang_9999@126.com;

zhouwenhao@fudan.edu.cn

${ }^{+}$Mingbang Wang, Deyi Zhuang and Mei Mei contributed equally to this

work.

${ }^{1}$ Shanghai Key Laboratory of Birth Defects, National Health Commision (NHC)

Key Laboratory of Neonatal Diseases, Division of Neonatology, National

Center for Children's Health, Children's Hospital of Fudan University,

Shanghai 201102, China

${ }^{4}$ Zhuhai Maternal and Children's Hospital, Zhuhai 519001, Guangdong, China

Full list of author information is available at the end of the article
}

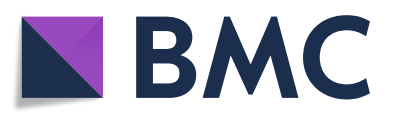

(c) The Author(s). 2020 Open Access This article is distributed under the terms of the Creative Commons Attribution 4.0 International License (http://creativecommons.org/licenses/by/4.0/), which permits unrestricted use, distribution, and

reproduction in any medium, provided you give appropriate credit to the original author(s) and the source, provide a link to the Creative Commons license, and indicate if changes were made. The Creative Commons Public Domain Dedication waiver (http://creativecommons.org/publicdomain/zero/1.0/) applies to the data made available in this article, unless otherwise stated. 


\section{Introduction}

Persistent pulmonary hypertension of the newborn (PPHN) affects 1.9 per thousand live births and is one of the important factors leading to neonatal mortality [1], the main feature of patients with PPHN is that pulmonary vascular resistance cannot be rapidly reduced to increase pulmonary blood flow and oxygen levels and to adapt to the postnatal environment [2]. Although PPHN can be rapidly diagnosed and treated with vasodilators and life support, the mortality rate remains high, at $8-10 \%$. In surviving patients, PPHN can cause neurological damage, cerebral palsy, deafness, blindness, and other complications [3, 4]. Sustained hypoxia resulting from PPHN has been associated with pulmonary vascular dysfunction, alveolar capillary dysplasia, severe lung dysplasia, and progressive lung injury [5]. Epidemiological data also support the association between PPHN and the development of lung disease $[5,6]$. Inhaled nitric oxide, a selective pulmonary vasodilator, is widely used in PPHN therapy; however, $30-40 \%$ of treated patients, particularly those with pulmonary parenchymal lesions and pulmonary hypoplasia, fail to achieve sustained improvement in oxygenation [7]. These findings suggest that there may be a genetic basis for the disease in some PPHN patients.

Recent studies have shown that genetic factors play an important role in the pathogenesis of pulmonary arterial hypertension (PAH) [8]. However, unlike PAH, PPHN is rarely familial and there has been little research on potential genetic associations [2]. We previously used target region sequencing (TRS) of genes associated with vascular activity in PPHN patients, and we found a significant association between the disease and a variant of the EDN1 endothelin 1-coding gene [9], recently, we screened clinically relevant mutations in children with PPHN patients through a target panel containing more than 2700 rare disease-related genes [10]. Niermeyer et al. found that the arterial oxygen saturation at birth and in the first 4 months after birth in Tibet (3658 $\mathrm{m}$ above sea level) was higher than that in Han newborns at plain, suggesting that genetic adaptation may allow sufficient oxygen to confer resistance to pulmonary hypertension [11]; at the same time, recent genome-wide studies have found that genetic basis of Tibetan population adapts to high-altitude chronic hypoxia, hypoxia-inducible factor pathway gene polymorphisms are significantly different among Tibetans and Han population [12].

In the present study, we sought to extend this study by screening for gene variants associated with PPHN in the high altitudes Tibetan population, which exhibits high adaptability to hypoxia and confers resistance to pulmonary hypertension. We first performed whole exome sequencing (WES) of 20 Tibetan PPHN patients and compared the results with a published dataset of 50 healthy Tibetan individuals to find PPHN-related single nucleotide polymorphisms (SNPs). We then validated in
80 patients from plain PPHN populations to see if the PPHN-related SNPs were independent of high altitudes.

\section{Materials and methods Patients}

The high altitudes PPHN patients were recruited from Lhasa People's Hospital, and the plain PPHN patients were recruited from Children's Hospital of Fudan University. PPHN was diagnosed by clinical and echocardiographic data, as detailed in our previous study [9]. Informed consent was obtained from a parent, and the study was approved by the ethics committee of the Children's Hospital of Fudan University (No. 2015-169).

\section{Whole exome and targeted region sequencing}

Genomic DNA was extracted from patient peripheral blood (1-2 ml) samples using a Magbind Blood DNA Kit (CW Biotech, Beijing, China) according to the manufacturer's instructions and was stored at $-20^{\circ} \mathrm{C}$. WES and TRS were performed as previously described $[9,13]$. In brief, whole exomes were captured using SeqCap EZ Exome (44 M) arrays (Roche, Basel, Switzerland). The targeted region was captured using a SeqCap Target Enrichment Kit (Roche) and sequenced using an Illumina HiSeq 2500 System (Illumina, San Diego, CA, USA) at BGI-Shenzhen (BGI, Shenzhen, China).

\section{Bioinformatics and statistical analysis Discovery stage}

For the case-control differential analysis, the raw WES datasets from 20 Tibetan PPHN patients were the cases and published WES datasets from 50 healthy Tibetan individuals [14] were the controls. First, all raw sequencing data were pre-processed using a customized bioinformatics pipeline as described previously [15], and clean reads were generated by removing adapters and filtering out low-quality reads with Trimmomatic [16]. Clean reads were then aligned to the human reference genome (Hg19) using the BWA-MEM algorithm [17] and further visualized using the SplicingViewer software [18], variants were called using GATK [19], and the variants were functionally annotated using ANNOVAR [20]. We then filtered out variants at depths of $<20$ in the PPHN samples and at depths of $<10$ in the controls, and Fisher's exact test was used to determine the difference between PPHN and controls. $P<0.001$ was considered significant. Genes with PPHN-associated variants were further enriched according to the KEGG pathway database (version 76 [21]). $P$ values were determined using Fisher's exact test, and $Q$ values were determined by falsediscovery rate correction of the $P$ values. $Q<0.05$ was considered significant. 


\section{Validation stage}

To validate the identified PPHN-associated variants in plain population, an additional 80 PPHN patients were sequenced. Variants at depths $<20$ were filtered out and published 800 healthy controls WES dataset [22] were used as controls for differences analysis using Fisher's exact test ( $\mathrm{P}$ value no more than 0.05 were considered significant). The association between gene variants and PPHN was predicted using Phenolyzer [23]. Nonsynonymous variants with allelic frequencies of $\leq 0.01$ in the Exome Aggregation Consortium (ExAC) database were considered potential disease-causing variants.

\section{Results}

A flowchart of the study design is shown in Fig. 1. Clinical information on the study participants is given in Additional file 1: Table S1. In the discovery stage, the average sequencing depth for the 20 Tibetan PPHN patients and 50 Tibetan controls were $40 \times$ and $20 \times$, respectively. After strictly filtering out variants with no or low-depth coverage (i.e., $<20$ and $<10$ for PPHN patients and controls, respectively), a total of 2023 variants were identified (Additional file 1: Table S2) and subjected to case-control differential analysis. Using Fisher's exact test for single-locus association analysis, a total of 166 significant differences were identified $(P<0.001$, Additional file 1: Table S3).

In a KEGG pathway analysis, we identified eight pathways enriched in genes with $\mathrm{PPHN}$-associated variants $(Q<0.05$, Table 1). These were Renal cell carcinoma, Salmonella infection, Pathways in cancer, Graft-versus-host disease, HIF-1 (hypoxia-inducible factor 1) signaling pathway, Bladder cancer, Regulation of actin cytoskeleton, and Gap junction. HIF-1 is a transcription factor that controls the expression of numerous hypoxia-related genes and plays a crucial role in cancer biology [24]. We found that three of the HIF-1 signaling pathway-associated genes; namely, EGF, EP300, and IL6, were also present in the other seven pathways enriched in PPHN-associated variants (Additional file 1: Table S4). Therefore, we turned our focus to genes in the HIF-1 signaling pathway. Interestingly, $49 \%(80 / 166)$ of the PPHN-associated variants were genes regulated by hypoxia or related to hypoxia tolerance

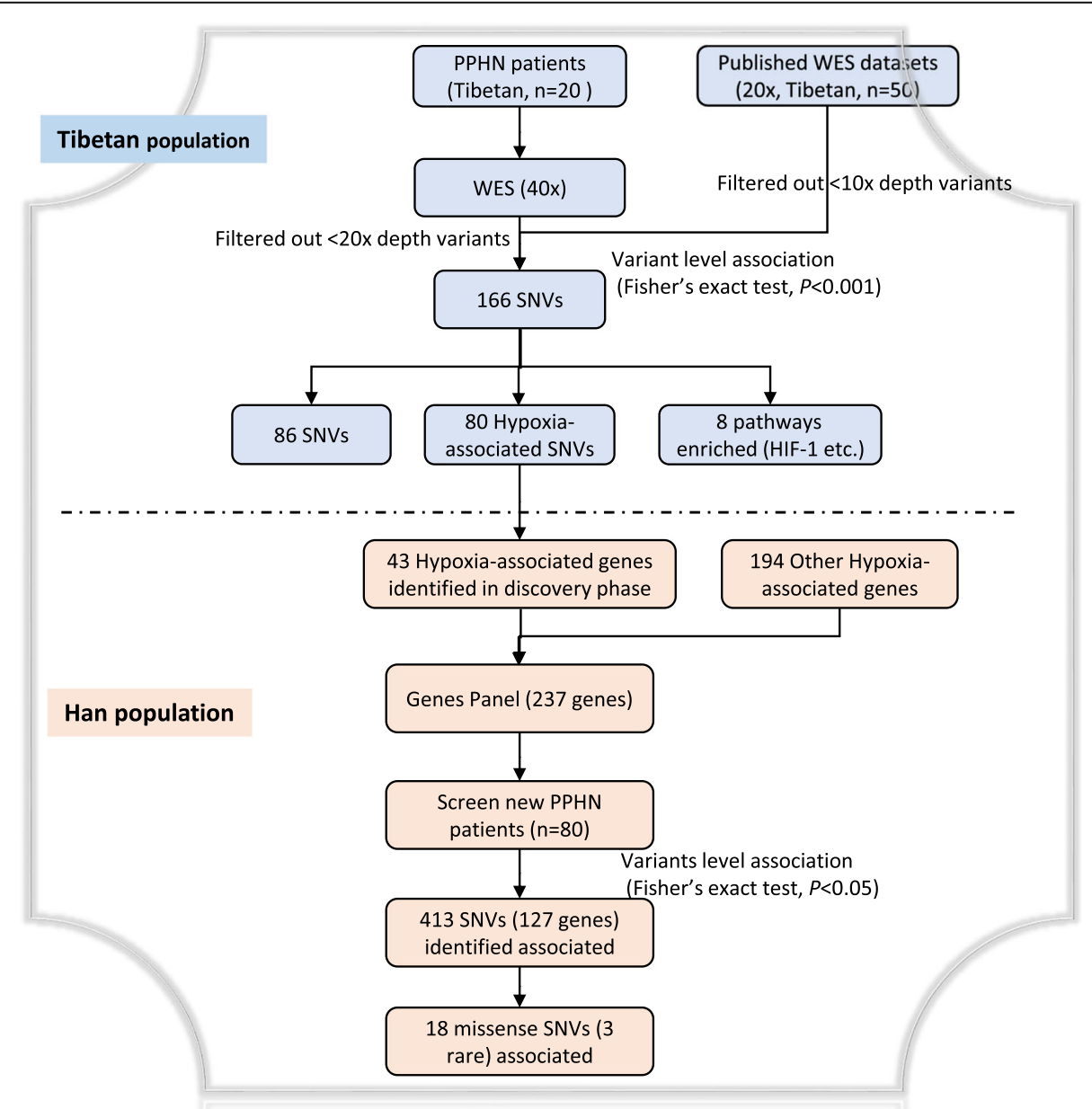

Fig. 1 Flowchart of the study design. HIF-1 = hypoxia-inducible factor-1; PPHN = persistent pulmonary hypertension of the newborn; SNVs= single nucleotide variants; WES = whole exome sequencing 
Table 1 Pathways enriched in PPHN-Associated gene variants identified in the tibetan population

\begin{tabular}{|c|c|c|c|c|c|}
\hline Pathway name & Pathway ID & PPHN genes (114) & All genes (17251) & $P$ value & Q value \\
\hline Renal cell carcinoma & ko05211 & 7 & 101 & $4.71 \mathrm{E}-06$ & 0.001 \\
\hline Salmonella infection & ko05132 & 10 & 317 & 4.79E-05 & 0.005 \\
\hline Pathways in cancer & ko05200 & 14 & 644 & 8.85E-05 & 0.006 \\
\hline Graft-versus-host disease & ko05332 & 5 & 74 & 0.0001293 & 0.006 \\
\hline HIF-1 signaling pathway & ko04066 & 7 & 185 & 0.0002281 & 0.009 \\
\hline Bladder cancer & ko05219 & 4 & 61 & 0.0007062 & 0.023 \\
\hline Regulation of actin cytoskeleton & ko04810 & 11 & 588 & 0.0017709 & 0.050 \\
\hline Gap junction & ko04540 & 5 & 135 & 0.0020232 & 0.050 \\
\hline
\end{tabular}

Definition of abbreviations: HIF-1 Hypoxia-inducible factor-1, PPHN Persistent pulmonary hypertension of the newborn

(Additional file 1: Table S5); we refer to these as hypoxiarelated genes.

To verify whether the correlation between hypoxiarelated associated genes and PPHN is independent of high altitude, we first conducted a systematic literature search and identified 246 hypoxia-related genes (Additional file 1: Table S5), and then designed a capture chip that captures the exon regions of 246 hypoxia-related genes, finally, the targeted region was sequenced in 80 plain PPHN patients with an average depth of 100x. As in the discovery stage, we use strict filtering standards to remove variants with no or low-depth coverage in the controls, and identified a total of 413 variants significantly associated with PPHN $(P<0.05$, Additional file 1 : Table S6) in 127 of the 246 hypoxia-related genes. Of these 413 , only $5 \%$ (21) were population-specific variants $(P<0.05$, Additional file 1: Table S7) and were not considered rare (minor allele frequency $[\mathrm{MAF}]>0.05$ in the ExAC database). Most of these variants (20/21) were located in the intronic or exonic regions and did not result in amino acid changes. One missense variant was associated with the FANCA (Fanconi anemia complementation group A) gene.

Eighteen of the PPHN-associated variants consisted of non-synonymous mutations in 14 genes; $A N P 32 D$, C12orf54, DR1, DUOXA1, FANCA, ITGAM, MBL2, MDH1B, PFKM, PLAU, PTX3, SIPA1L2, TMEM206, and TTLL3 (Table 2). Most of these variants (15/18) were not rare $(\mathrm{MAF}>0.01)$ in ExAC. The three rare $(\mathrm{MAF}<$ 0.01 ) variants were two missense mutations in TTLL3

Table 2 PPHN-Associated missense variants identified in the han population

\begin{tabular}{|c|c|c|c|c|c|c|c|c|c|c|}
\hline Chr & Pos & Ref & Alt & Variants & Gene & PPHN (MAF) & Ctrl (MAF) & $P$ value & OR & $\mathrm{MAF}^{\mathrm{a}}$ \\
\hline chr12 & $48,866,585$ & A & C & p.L46F & ANP32D & 0.604 & 0.687 & 0.0487 & 0.69 & 0.187 \\
\hline chr12 & $48,888,594$ & C & $\mathrm{T}$ & p.P86S & C12orf54 & 0.188 & 0.12 & 0.0265 & 1.70 & 0.372 \\
\hline chr1 & $93,826,178$ & A & $\mathrm{T}$ & p.E171D & $D R 1$ & 0.052 & 0.016 & 0.0121 & 3.31 & 0.092 \\
\hline chr15 & $45,409,732$ & C & G & p.R433P & DUOXA1 & 0.071 & 0.033 & 0.0365 & 2.28 & 0.083 \\
\hline chr16 & $89,857,935$ & G & A & p.A412V & FANCA & 0.156 & 0.29 & 0.0005 & 0.45 & 0.065 \\
\hline chr16 & $89,805,914$ & T & C & p.T1328A & FANCA & 0.156 & 0.274 & 0.0017 & 0.49 & 0.031 \\
\hline chr16 & $89,839,766$ & G & C & p.P643A & FANCA & 0.182 & 0.297 & 0.0032 & 0.53 & 0.025 \\
\hline chr16 & $89,836,323$ & C & $\mathrm{T}$ & p.G809D & FANCA & 0.968 & 0.99 & 0.0443 & 0.30 & 0.467 \\
\hline chr16 & $31,341,863$ & G & C & p.E477D & ITGAM & 0.026 & 0.005 & 0.027 & 5.29 & 0.001 \\
\hline chr10 & $54,531,235$ & C & $\mathrm{T}$ & p.G54D & MBL2 & 0.13 & 0.209 & 0.0262 & 0.57 & 0.139 \\
\hline chr2 & $207,603,221$ & $\mathrm{~T}$ & C & p.T515A & MDH1B & 0.052 & 0.021 & 0.0475 & 2.52 & 0.087 \\
\hline chr12 & $48,501,161$ & A & $\mathrm{T}$ & p.H2L & PFKM & 0.532 & 0.621 & 0.0473 & 0.70 & 0.266 \\
\hline chr10 & $75,673,101$ & $\mathrm{~T}$ & C & p.L105P & PLAU & 0.558 & 0.682 & 0.0038 & 0.59 & 0.755 \\
\hline chr3 & $157,155,314$ & C & A & p.A48D & PTX3 & 0.857 & 0.775 & 0.0234 & 1.74 & 0.625 \\
\hline chr1 & $232,539,219$ & C & $\mathrm{T}$ & p.G695S & SIPATL2 & 0.442 & 0.544 & 0.0218 & 0.66 & 0.065 \\
\hline chr1 & $212,587,320$ & T & C & p.N47D & TMEM206 & 0.162 & 0.088 & 0.0076 & 2.02 & 0.035 \\
\hline chr3 & $9,876,568$ & C & $\mathrm{T}$ & p.P777S & $\pi T L 3$ & 0.026 & 0.005 & 0.027 & 5.29 & 0.001 \\
\hline chr3 & $9,860,595$ & G & A & p.E317K & $\pi T L 3$ & 0.006 & 0.038 & 0.0463 & 0.17 & 0.001 \\
\hline
\end{tabular}

${ }^{a}$ Minor allele frequency (MAF) according to the Exome Aggregation Consortium database. Definition of abbreviations: Alt Alteration, Chr Chromosome, Ctrl Control Pos Position, OR Odds ratio, PPHN Persistent pulmonary hypertension of the newborn, Ref Reference 
(tubulin tyrosine ligase-like 3), p.E317K and p.P777S, and one missense mutation in ITGAM (integrin subunit alpha M), p.E1071D. Both TTLL3 and ITGAM were predicted to be associated with the PPHN phenotype using Phenolyzer $(P=0.002589$ and $P=0.03325$, respectively).

\section{Discussion}

In this two-stage study, we first compared WES data from 20 Tibetan PPHN patients and 50 healthy Tibetan controls living at high altitudes and identified 166 PPHNassociated variants, $49 \%$ of which were derived from 43 hypoxia-related genes; considering many studies have shown that the differences in the genetic background between Tibet and Han are characterized by hypoxia-related genetic polymorphisms [12]; to further verify whether the association of hypoxia-associated variants with PPHN is independent of high altitude life, we performed a targeted validation study of hypoxia-related genes in an additional 80 Han PPHN patients living at low altitudes. Finally, we discovered 413 PPHN-associated variants from 127 hypoxia-related genes in Han population.

Significant changes in blood coagulation at high altitudes may predispose individuals to pulmonary hypertension [25, 26]. Negi et al. [27] conducted a cross-sectional survey of permanent inhabitants in the Spiti Valley at an altitude of 3000 to $4200 \mathrm{~m}$, although no significant correlation was found between altitude and pulmonary hypertension. However, it is still found that the prevalence rate is increasing with the increase of altitude; the data published to date have not been identified in plateau populations as genetic susceptibility to individual pulmonary hypertension $[28,29]$. Our results indicates that the association of hypoxia-related genes with PPHN does not depend on high-altitude life, at the same time, some rare mutations associated with PPHN were also found.

Two rare missense mutations identified in TTLL3 (p.E317K and p.P777S) were associated with PPHN. TTLL3 catalyzes the ATP-dependent restoration of tyrosine to the C-terminus of $\alpha$-tubulin, which polymerizes into microtubules [30, 31]. Microtubule proliferation has been found in the right ventricular myocytes of rats with monocrotalineinduced pulmonary hypertension, accompanied by upregulated expression of $\alpha$ - and $\beta$-tubulin [32]. The detyrosination/tyrosination cycle of tubulin is important for regulating the mechanical stabilization of long-lived microtubules in cells, and abnormalities in this cycle are involved in the development of hypertension [33]. A recent study found that TTLL3 can also ligate various unnatural amino acids to the C-terminus of tubulin [34]. The crystal structure of TTLL3 has recently been solved and suggests that TTLL3 can ligate glycine and glutamate to the tubulin Cterminus, a process crucial for the biogenesis and stability of microtubules in cilia, flagella, spindles, neuronal processes, and platelets $[35,36]$. Glutamylation, the most prevalent post-translational modification of tubulin, stabilizes microtubules and regulates the recruitment and activity of microtubule-interacting proteins [37]. Valenstein et al. found that glutamylation was a major regulator of hereditary spastic paraplegia [38]. Consistent with the potential importance of microtubule-associated proteins in PPHN, we found significant enrichment of genes associated with regulation of the actin cytoskeleton among the $166 \mathrm{PPHN}$ associated variants identified in the Tibetan population. These included TTLL3, EGF, GOLGA1, KRAS, IQGAP1, LRRFIP2, RB1CC1, TEX14, GCOM1, ARPC4-TTLL3, and $M Y Z A P$. Interestingly, Fediuk et al. found that the eicosanoid thromboxane can induce actin polymerization in hypoxic neonatal pulmonary arterial myocytes [39, 40].

Rare missense mutation identified in in ITGAM (p.E1071D) was associated with PPHN, ITGAM encodes the integrin alpha $M$ chain, also known as Mac-1, CD11b/ CD18, or CR3A. Integrins play important roles in cell-cell adhesion by mediating transmembrane connections to the cytoskeleton and activating intracellular signaling pathways. A previous study showed that integrins are differentially regulated in pulmonary artery smooth muscle cells during pulmonary hypertension [41]. In addition, pulmonary artery endothelial cells from patients with idiopathic pulmonary hypertension show decreased adhesion to laminin, and loss of interaction between $\alpha 3$ integrins and the tumor promoter APC (adenomatous poliposis coli) promotes endothelial apoptosis in mice and humans [42]. Furthermore, a recent study showed that induced pluripotent stem cell-derived endothelial cells from a patient with familial PAH showed reduced adhesion compared with control cells [43]. The missense mutation identified in the present study, p.E1071D, lies in the C-terminal domain of ITGAM, which may be responsible for recognizing short peptide motifs [44] that are involved in integrin-extracellular matrix interactions. This suggests a potential mechanism by which aberrant behavior of the ITGAM variant may contribute to PPHN.

Nevertheless, it is unclear how this integrin may be involved in PAH or PPHN or whether the ITGAM mutation is protective or a risk factor. Jiang et al. found that platelet-mediated mesenchymal stem cell homing to the lung can reduce monocrotaline-induced rat pulmonary hypertension [45]. A recent study showed that ITGAM can regulate thrombosis via interaction with platelet GPIb [46], suggesting that it may play a protective function against thrombosis. However, upregulation of integrins may also be associated with the onset of PPHN, as previous studies have shown that integrins $\alpha v \beta 6$ and $\alpha v \beta 8$ are upregulated in bronchial epithelial cells from patients with systemic sclerosis, a disease often associated with PAH [47]. Welschoff et al. found that the tripeptide Arg-Gly-Asp, which inhibits the adhesion of several integrins, can induce relaxation of 
pulmonary arteries and reduce pulmonary arterial pressure [48].

PPHN is not a single disease, but a clinical syndrome caused by multiple factors, that may present with different clinical conditions requiring different treatments and with variable outcomes. The PPHN related genetic variants we described could improve the understanding of the pathogenesis of PPHN and, consequently, increase the spectrum of available treatments by targeting hypoxia related gene pathways $[49,50]$. Finally our paper may also suggest the feasibility of whole-genome sequencing for the screening of clinically relevant mutations, associated with PPHN.

This study has some innovations. To the best of our knowledge, we have performed exome screening for the first time in PPHN patients; we found that hypoxia-related genes are associated with PPHN and are not dependent on high altitudes. It provides a genetic basis for the pathogenesis of PPHN and also provides a target for our PPHN genetic screening in newborns. There are some limitations to our study. The published Tibetan WES datasets used as controls were of low coverage, and the rigorous filtering may have eliminated Tibetan-specific variants associated with PPHN. Although we have identified three rare missense variants associated with PPHN, further functional studies will be necessary to understand how they contribute to the pathogenesis of PPHN.

\section{Conclusions}

We conducted a two-stage genetic study of 100 PPHN patients and identified several hypoxia-associated gene variants that may be associated with PPHN, and the association of hypoxia-associated gene mutations with PPHN does not depend on high altitude life.

\section{Supplementary information}

Supplementary information accompanies this paper at https://doi.org/10. 1186/s12931-020-1314-5.

Additional file 1: Table S1. Clinical information on study participants. Table S2. List of 2023 variants used for case-control analysis in Tibetan population. Table S3. List of 166 gene variants significantly associated with PPHN $(P<0.001)$ identified in the Tibetan population. Table S4. List of pathways enriched in the 166 variants identified in the Tibetan population. Table S5. List of 237 hypoxia-related genes studied in the Han population. Table S6. List of 413 variants significantly associated with PPHN $(P<0.05)$ identified in the Han population. Table S7. List of 21 population-specific variants significantly associated with PPHN $(P<$ 0.05).

\section{Abbreviations}

ExAC: Exome Aggregation Consortium; KEGG: Kyoto Encyclopedia of Genes and Genomes; PAH: Pulmonary arterial hypertension; PPHN: Persistent pulmonary hypertension of the newborn; TRS: Target region sequencing; WES: Whole exome sequencing

\section{Acknowledgements}

Thanks to Dr. Yulan Lu from Fudan University, and Dr. Jinyu Wu from the Beijing Institute of Life Sciences, Chinese Academy of Sciences for their assistance in information analysis.

\section{Authors' contributions}

MW, GL and WZ conceptualized and designed the study, supervised data collection and revised manuscripts, DZ, MM, HM and GC completed sample collection and clinical evaluation, MW drafted manuscripts, reviewed and revised manuscripts, and FH and ZL conducted bioinformatics analysis. All authors read and approved the final manuscript.

\section{Funding}

This project was supported by National Natural Science Foundation of China (Program No. 81701351, No. 81701498 and No.81720108018), Shanghai Municipal Commission of Health and Family Planning (GDEK201701), by Shanghai Municipal Science and Technology Major Project (2018SHZDZX05), and by Super Postdoctoral Program of Fudan University.

\section{Availability of data and materials}

The datasets for this study can be found in the supplemental material, and the raw data can be obtained from the corresponding author under request.

\section{Ethics approval and consent to participate}

The study obtained the informed consent of the participant's guardian, was approved by the Ethics Committee of Children's Hospital of Fudan University (No. 2015-169), and complied with the Helsinki Declaration.

\section{Consent for publication}

All authors agree to publish this manuscript.

\section{Competing interests}

The authors declare that they have no competing interests.

\section{Author details}

'Shanghai Key Laboratory of Birth Defects, National Health Commision (NHC) Key Laboratory of Neonatal Diseases, Division of Neonatology, National Center for Children's Health, Children's Hospital of Fudan University, Shanghai 201102, China. ${ }^{2}$ Xiamen Key Laboratory of Neonatal Diseases, Neonatal Medical Center, Xiamen Children's Hospital, Children's Hospital of Fudan University (Xiamen Branch), Xiamen 361006, Fujian, China. ${ }^{3}$ Division of Pulmonology, Children's Hospital of Fudan University, Shanghai 201102, China.

${ }^{4}$ Zhuhai Maternal and Children's Hospital, Zhuhai 519001, Guangdong, China. ${ }^{5}$ Department of Population and Quantitative Health Sciences, University of Massachusetts Medical School, Worcester, MA 01655, USA. IImunobio, Shenzhen, Guangdong, China. 'Division of Neonatology, Children's Hospital of Fudan University, Shanghai 201102, China. 'Key Laboratory of Birth Defects, Children's Hospital of Fudan University, Shanghai 200436, China.

Received: 18 September 2019 Accepted: 4 February 2020

Published online: 13 February 2020

\section{References}

1. Walsh-Sukys MC, Tyson JE, Wright LL, Bauer CR, Korones SB, Stevenson DK, Verter J, Stoll BJ, Lemons JA, Papile LA, et al. Persistent pulmonary hypertension of the newborn in the era before nitric oxide: practice variation and outcomes. Pediatrics. 2000;105:14-20.

2. Goncharova EA, Gladwin MT, Kawut SM. Update in pulmonary vascular diseases 2014. Am J Respir Crit Care Med. 2015;192:544-50.

3. Porta NF, Steinhorn RH. Pulmonary vasodilator therapy in the NICU: inhaled nitric oxide, sildenafil, and other pulmonary vasodilating agents. Clin Perinatol. 2012:39:149-64.

4. Konduri GG, Vohr B, Robertson C, Sokol GM, Solimano A, Singer J, Ehrenkranz RA, Singhal N, Wright LL, Van Meurs K, et al. Early inhaled nitric oxide therapy for term and near-term newborn infants with hypoxic respiratory failure: neurodevelopmental follow-up. J Pediatr. 2007;150:235-40 240 e231.

5. Bishop NB, Stankiewicz P, Steinhorn RH. Alveolar capillary dysplasia. Am J Respir Crit Care Med. 2011;184:172-9.

6. Weijerman ME, van Furth $A M$, van der Mooren MD, van Weissenbruch MM, Rammeloo L, Broers CJ, Gemke RJ. Prevalence of congenital heart defects and persistent pulmonary hypertension of the neonate with Down syndrome. Eur J Pediatr. 2010;169:1195-9.

7. Gonzalez A, Fabres J, D'Apremont I, Urcelay G, Avaca M, Gandolfi C, Kattan J. Randomized controlled trial of early compared with delayed use of inhaled nitric oxide in newborns with a moderate respiratory failure and pulmonary hypertension. J Perinatol. 2010;30:420-4. 
8. Austin ED, Loyd JE. The genetics of pulmonary arterial hypertension. Circ Res. 2014;115:189-202.

9. Mei M, Cheng G, Sun B, Yang L, Wang H, Sun J, Zhou W. EDN1 gene variant is associated with neonatal persistent pulmonary hypertension. Sci Rep. 2016;6:29877.

10. Liu X, Mei M, Chen X, Lu Y, Dong X, Hu L, Hu X, Cheng G, Cao Y, Yang L, Zhou W. Identification of genetic factors underlying persistent pulmonary hypertension of newborns in a cohort of Chinese neonates. Respir Res. 2019;20:174.

11. Niermeyer S, Yang P, Shanmina D, Zhuang J, Moore LG. Arterial oxygen saturation in Tibetan and Han infants born in Lhasa, Tibet. N Engl J Med. 1995:333:1248-52.

12. Bigham AW, Lee FS. Human high-altitude adaptation: forward genetics meets the HIF pathway. Genes Dev. 2014;28:2189-204.

13. Zhou J, Yang F, Li X, Zhou S, He F, Liang J, Wang Y, Wang M, Xu R. Whole Exome Sequencing Revealed Clinical Relevant Variants in A Family Affected with Autism Spectrum Disorder. J Psychiatry Brain Sci. 2016;1:3.

14. Yi X, Liang Y, Huerta-Sanchez E, Jin X, Cuo ZX, Pool JE, Xu X, Jiang H, Vinckenbosch $N$, Korneliussen TS, et al. Sequencing of 50 human exomes reveals adaptation to high altitude. Science. 2010;329:75-8.

15. Wang T, Liu Q, Li X, Wang X, Li J, Zhu X, Sun ZS, Wu J. RRBS-analyser: a comprehensive web server for reduced representation bisulfite sequencing data analysis. Hum Mutat. 2013;34:1606-10.

16. Bolger AM, Lohse M, Usadel B. Trimmomatic: a flexible trimmer for Illumina sequence data. Bioinformatics. 2014;30:2114-20.

17. Li H. Toward better understanding of artifacts in variant calling from highcoverage samples. Bioinformatics. 2014;30:2843-51.

18. Liu Q, Chen C, Shen E, Zhao F, Sun Z, Wu J. Detection, annotation and visualization of alternative splicing from RNA-Seq data with SplicingViewer. Genomics. 2012;99:178-82.

19. McKenna A, Hanna M, Banks E, Sivachenko A, Cibulskis K, Kernytsky A, Garimella K, Altshuler D, Gabriel S, Daly M, DePristo MA. The genome analysis toolkit: a MapReduce framework for analyzing next-generation DNA sequencing data. Genome Res. 2010;20:1297-303.

20. Wang K, Li M, Hakonarson H. ANNOVAR: functional annotation of genetic variants from high-throughput sequencing data. Nucleic Acids Res. 2010;38:e164.

21. Ogata H, Goto S, Sato K, Fujibuchi W, Bono H, Kanehisa M. KEGG: Kyoto encyclopedia of genes and genomes. Nucleic Acids Res. 1999;27:29-34.

22. Tang H, Jin X, Li Y, Jiang H, Tang X, Yang X, Cheng H, Qiu Y, Chen G, Mei J, et al. A large-scale screen for coding variants predisposing to psoriasis. Nat Genet. 2014;46:45-50.

23. Yang $H$, Robinson PN, Wang K. Phenolyzer: phenotype-based prioritization of candidate genes for human diseases. Nat Methods. 2015;12:841-3.

24. Semenza GL. Targeting HIF-1 for cancer therapy. Nat Rev Cancer. 2003;3:721-32.

25. Singh I, Chohan IS. Blood coagulation changes at high altitude predisposing to pulmonary hypertension. Br Heart J. 1972;34:611-7.

26. Scherrer U, Turini P, Thalmann S, Hutter D, Salmon CS, Stuber T, Shaw S, Jayet PY, Sartori-Cucchial C, Villena M, et al. Pulmonary hypertension in high-altitude dwellers: novel mechanisms, unsuspected predisposing factors. Adv Exp Med Biol. 2006;588:277-91.

27. Negi PC, Marwaha R, Asotra S, Kandoria A, Ganju N, Sharma R, Kumar RV, Bhardwaj R. Prevalence of high altitude pulmonary hypertension among the natives of Spiti Valley--a high altitude region in Himachal Pradesh, India. High Alt Med Biol. 2014;15:504-10.

28. Mirrakhimov AE, Strohl KP. High-altitude pulmonary hypertension: an update on disease pathogenesis and management. Open Cardiovasc Med J. 2016;10:19-27.

29. Xu XQ, Jing ZC. High-altitude pulmonary hypertension. Eur Respir Rev. 2009; 18:13-7.

30. Erck C, Frank R, Wehland J. Tubulin-tyrosine ligase, a long-lasting enigma. Neurochem Res. 2000;25:5-10.

31. Rogowski K, Juge F, van Dijk J, Wloga D, Strub JM, Levilliers N, Thomas D, Bre MH, Van Dorsselaer A, Gaertig J, Janke C. Evolutionary divergence of enzymatic mechanisms for posttranslational polyglycylation. Cell. 2009;137:1076-87.

32. Stones R, Benoist D, Peckham M, White E. Microtubule proliferation in right ventricular myocytes of rats with monocrotaline-induced pulmonary hypertension. J Mol Cell Cardiol. 2013;56:91-6.

33. Xu Z, Schaedel L, Portran D, Aguilar A, Gaillard J, Marinkovich MP, Thery M, Nachury MV. Microtubules acquire resistance from mechanical breakage through intralumenal acetylation. Science. 2017;356:328-32.

34. Schumacher D, Lemke O, Helma J, Gerszonowicz L, Waller V, Stoschek T, Durkin PM, Budisa N, Leonhardt H, Keller BG, Hackenberger CPR. Broad substrate tolerance of tubulin tyrosine ligase enables one-step site-specific enzymatic protein labeling. Chem Sci. 2017;8:3471-8.
35. Garnham CP, Yu I, Li Y, Roll-Mecak A. Crystal structure of tubulin tyrosine ligase-like 3 reveals essential architectural elements unique to tubulin monoglycylases. Proc Natl Acad Sci U S A. 2017;114:6545-50.

36. Yu I, Garnham CP, Roll-Mecak A. Writing and Reading the tubulin code. J Biol Chem. 2015;290:17163-72.

37. Garnham CP, Vemu A, Wilson-Kubalek EM, Yu I, Szyk A, Lander GC, Milligan RA, Roll-Mecak A. Multivalent microtubule recognition by tubulin tyrosine ligase-like family Glutamylases. Cell. 2015;161:1112-23.

38. Valenstein ML, Roll-Mecak A. Graded control of microtubule severing by tubulin Glutamylation. Cell. 2016;164:911-21.

39. Fediuk J, Sikarwar AS, Nolette N, Dakshinamurti S. Thromboxane-induced actin polymerization in hypoxic neonatal pulmonary arterial myocytes involves Cdc42 signaling. Am J Phys Lung Cell Mol Phys. 2014;307:L877-87.

40. Fediuk J, Gutsol A, Nolette N, Dakshinamurti S. Thromboxane-induced actin polymerization in hypoxic pulmonary artery is independent of rho. Am J Phys Lung Cell Mol Phys. 2012;302:L13-26.

41. Umesh A, Paudel O, Cao YN, Myers AC, Sham JS. Alteration of pulmonary artery integrin levels in chronic hypoxia and monocrotaline-induced pulmonary hypertension. J Vasc Res. 2011;48:525-37.

42. de Jesus Perez VA, Yuan K, Orcholski ME, Sawada H, Zhao M, Li CG, Tojais NF, Nickel N, Rajagopalan V, Spiekerkoetter E, et al. Loss of adenomatous poliposis coli-alpha3 integrin interaction promotes endothelial apoptosis in mice and humans. Circ Res. 2012;111:1551-64.

43. Gu M, Shao NY, Sa S, Li D, Termglinchan V, Ameen M, Karakikes I, Sosa G, Grubert F, Lee J, et al. Patient-specific iPSC-derived endothelial cells uncover pathways that protect against pulmonary hypertension in BMPR2 mutation carriers. Cell Stem Cell. 2017;20:490-504 e495.

44. Xiao T, Takagi J, Coller BS, Wang JH, Springer TA. Structural basis for allostery in integrins and binding to fibrinogen-mimetic therapeutics. Nature. 2004;432:59-67.

45. Jiang $L$, Song $X H$, Liu $P$, Zeng $C L$, Huang $Z S$, Zhu $L$, Jiang $Y Z$, Ouyang $H W$, $\mathrm{Hu} \mathrm{H}$. Platelet-mediated mesenchymal stem cells homing to the lung reduces monocrotaline-induced rat pulmonary hypertension. Cell Transplant. 2012;21:1463-75.

46. Wang Y, Gao H, Shi C, Erhardt PW, Pavlovsky A, AS D, Bledzka K, Ustinov V, Zhu L, Qin J, et al. Leukocyte integrin mac-1 regulates thrombosis via interaction with platelet GPIbalpha. Nat Commun. 2017:8:15559.

47. Lafyatis R. Transforming growth factor beta--at the Centre of systemic sclerosis. Nat Rev Rheumatol. 2014:10:706-19.

48. Welschoff J, Matthey M, Wenzel D. RGD peptides induce relaxation of pulmonary arteries and airways via beta3-integrins. FASEB J. 2014;28:2281-92.

49. Zhang T, Kawaguchi N, Yoshihara K, Hayama E, Furutani Y, Kawaguchi K, Tanaka T, Nakanishi T. Silibinin efficacy in a rat model of pulmonary arterial hypertension using monocrotaline and chronic hypoxia. Respir Res. 2019;20:79.

50. Li N, Guan Y, Tian YM, Ma HJ, Zhang X, Zhang Y, Wang S. Chronic intermittent hypobaric hypoxia ameliorates renal vascular hypertension through upregulating NOS in nucleus Tractus Solitarii. Neurosci Bull. 2019;35:79-90.

\section{Publisher's Note}

Springer Nature remains neutral with regard to jurisdictional claims in published maps and institutional affiliations.

Ready to submit your research? Choose BMC and benefit from:

- fast, convenient online submission

- thorough peer review by experienced researchers in your field

- rapid publication on acceptance

- support for research data, including large and complex data types

- gold Open Access which fosters wider collaboration and increased citations

- maximum visibility for your research: over $100 \mathrm{M}$ website views per year

At $\mathrm{BMC}$, research is always in progress.

Learn more biomedcentral.com/submissions 